\title{
A Web-Based Yorùbá to English Bilingual Lexicon for Building Technicians
}

\author{
Abiola O.B ${ }^{1}$, Adeyemo O.A ${ }^{2}$, Saka-Balogun O.Y ${ }^{3}$, Okesola F. ${ }^{4}$ \\ ${ }^{1}$ Afe Babalola University, Ado-Ekiti, Nigeria, abiolatoyinbunmi@ gmail.com \\ ${ }^{2}$ Afe Babalola University, Ado-Ekiti, Nigeria, adeyemo@abuad.edu.ng \\ ${ }^{3}$ Afe Babalola University, Ado-Ekiti, Nigeria, balogunld@ abuad.edu.ng \\ ${ }^{4}$ Afe Babalola University, Ado-Ekiti, Nigeria, abiolaob@abuad.edu.ng
}

\begin{abstract}
The importance of language and communication cannot be overemphasized. In Nigeria, the dominance of the English language as a medium of communication in various sectors: education, judiciary, government etc. is alarming, as the major indigenous languages are gradually moving towards extinction. A Yorùbá to English bilingual lexicon for building technicians has been developed in this research work to reduce communication gaps, create an interactive platform for learning and enhance business transactions among building technicians. The implementation of the architecture is based on the direct approach to machine translations. The programming language tools used were ASP. NET C\#, HTML, CSS, JavaScript and BOOTSTRAP as front end to provide a user friendly interface and Microsoft SQL (Structure Query Language) Server as backend to create the database for the data gathered. The system can be used by building technicians and all who are willing to learn basic terminologies from Yorùbá to English under the subject domain.
\end{abstract}

Key Words: Bilingual Lexicon, Building Technicians, Direct-Based, Machine Translation.

\section{INTRODUCTION}

Artificial Intelligence is expanding rapidly and since its inception, the study of natural language processing (NLP) also known as human language processing has been an essential and paramount area of research in the field. Natural language processing (NLP) has been defined in a general way as a discipline whose ultimate goal is to enable people to interact with machines using their human faculties and skills. In order words, machines should be able to understand spoken or written sentences constructed according to the rules of some human languages and should be capable of generating in reply meaningful sentences in such languages [14].

Natural language processing (NLP) is also defined as an area of research and application that gives machines the ability to read and understand the languages spoken by humans. In other words, it is a term that is used to distinguish human languages such as English, Yorùbá, Spanish, Swahili,
Swedish, French, Arabic etc. from formal or computer languages such as Python, C++, Java or LISP. In the philosophy of language, a natural language is also known as an ordinary language that is spoken, written, or signed by humans for general purpose communication as distinguished from computer programming languages [4], [1].

Natural language processing systems take strings of words (sentences) as their inputs and produce structured representations capturing the meaning of those strings as their outputs. NLP provides both theory and implementations for a range of application areas which include the following among others: spelling and grammar, checking and suggesting alternatives for the errors, word prediction, Information retrieval, text categorization, summarization, question answering, Information extraction, machine translation, sentiment analysis, optical character recognition, speech recognition and processing, speech synthesis, spoken dialogue system among others [12].

\section{Machine Translation}

Machine Translation (MT) is a subfield under artificial intelligence that mainly deals with the transformation from one human language to another. One of the important goals of computational linguistics is a fully automatic machine translation between human languages therefore; machine translation is the application of computers to the task of translating texts from one human language to another. When an entire document is given to the computer system for automatic translation from one language source language (SL) to another language target language (TL), it is known as machine translations [16], [19].

The various modes of machine translations are: text-to-text (T2T), text-to-speech (T2S), speech-to-text (S2T) and speech-to-speech (S2S). Attempts of language translations are as old as computers themselves because language is a powerful tool of control which forms a large part of the culture of people and learning a language is not only reaching out to others but to maintain a variety of social bond and shared sense of values [20].

\section{Machine Translations Approaches}

Fully automated machine translation is the aim of a good translator. The idea is to feed in the text to be translated and 
get the text out in another language. Unfortunately, the implementation of this idea has been presenting obstacles that have not been overcome due to the complexity of some languages. Many approaches have been used in recent times and in literatures to develop machine translation systems. Each of these approaches has its own advantages and challenges or drawbacks. A fully automated machine translation system is classified according to its main paradigm and distinctive characteristics based on the resources used. There are three main paradigms of a fully automated machine translation system. The rule-based approach which is sub-divided into the direct, transfer and Interlingua; the empirical system which is sub-divided into statistical-based (SMT), example-based (EBMT) and neural machine translation systems (NMT) respectively. The hybrid-based approach which comprises of the single engine hybridization (SEH) and multiple engine hybridization (MEH).

\section{a. The Rule-Based Approach}

In the rule-based approach to machine translations, human experts specify a set of rules to describe a translation process. An enormous and extensive amount of inputs from human experts is required in this system as it involves the application of morphological, syntactic and semantic rules in the analysis of the source language texts and the synthesis of the target-language texts. In this approach, a database of translation rules is used to translate text from source to target language respectively. The rule-based approach deals with word-order problem and since it uses linguistic knowledge, any produced errors can be traced [13], [18]. The rule-based approach is sub-divided into the direct, transfer and Interlingua:

\section{The Direct-Based Approach (DBMT)}

The direct-based approach (DBMT) is the primitive or the original approach which involves the replacement of the words of the source language with words of the target language without much linguistic analysis and processing. The major resource used by this approach is a bilingual dictionary or lexicon and this is why it is known as dictionary-driven approach. It involves simple word for word translation of texts. The DBMT system starts with morphological analysis which removes morphological inflections from the words to get the root word from the source language words, after which the bilingual dictionary lookup is done in which a bilingual dictionary is looked up to get the target-language equivalents of source words. This approach is designed for unidirectional translation between one pair of languages [5], [15], [16].

\section{The Interlingua-Based Approach:}

In the Interlingua approach, the source language text to be translated is transformed into an Interlingua language, which is a neutral representation that is independent of any language. The target language is then generated from the Interlingua language [11].

\section{The Transfer-Based Approach:}

The transfer-based approach to machine translation is similar to the Interlingua machine translation in that, it creates a translation from an intermediate representation that simulates the meaning of the original sentence. However, unlike Interlingua MT, it depends partially on the language pair involved in the translation. On the basis of the structural differences between the source and target language, there are three major modules or phases in the transfer-based approach: the analysis phase where the source language text is transformed into abstract source language representation and the linguistic information about the sentence structure and each word in the text is obtained; the transfer phase where the source to target text transfer is carried out with the help of a bilingual dictionary which is stored in the transfer module for easy accessibility when the system is running; and the synthesis or generation phase where the target language representation is re-organized and cleaned [13].

\section{b. The Empirical Systems}

The empirical system is divided into three main approaches: the statistical-based machine translation (SMT), the example-based machine translation (EBMT) and the neural machine translation (NMT).

\section{The Statistical-Based System}

The EBMT and the SMT rely on parallel data for their analysis and they are usually referred to as corpus-based approaches respectively. In the corpus-based approach, knowledge is automatically extracted by analyzing translation examples from a parallel corpus built by human experts. SMT requires less human efforts to undertake translation and it is characterized by the use of machine learning techniques. It is a machine translation paradigm where translations are generated on the basis of statistical models. The statistical-based system is further divided into phrase-based (PBSMT), hierarchical-based (HBSMT) and syntax-based (SBSMT) respectively [11].

\section{The Example-Based System (EBMT)}

In this approach, new translations are formed on the basis of the previously compiled translations. The main idea behind EBMT is translation by analogy, as it makes use of past translation examples to generate the translation of a given input. EBMT systems use variety of linguistic resources such as dictionaries to translate text, and a bilingual corpus as its main knowledge-base at run-time [11].

\section{Neural Machine Translation (NMT)}

Neural machine translation (NMT) is an approach to machine translation that uses a large artificial neural network to predict the likelihood of a sequence of words, typically modeling entire sentences in a single integral model. Deep neural machine translation is an extension of neural machine translation and they both use a large neural network with the difference that deep NMT processes multiple neural network layers instead of just one. Unlike the conventional translation 
systems, all parts of the neural machine translation model are trained jointly (end-to-end) to maximize the translation performance [11].

\section{c. The Hybrid-Based Approach (HMT)}

The hybrid approach leverages the strengths of two or more major approaches by combining their features. It is a method of machine translation that is characterized by the use of multiple machine translation approaches within a single machine translation system. The main interest of hybrid systems is to improve the quality of machine translation in terms of fluency and adequacy. There are several popular machine translation systems which employ hybrid methods. Examples are: PROMT, SYSTRAN and Asia Online. The hybrid technique is useful at compensating the weak points of an approach with the strong points of another one. Hybrid systems can be categorized into two major groups: Single-Engine Hybridization (SEH) and Multi-Engine Hybridization (MEH) [7].

\section{Motivation}

Language is an efficient and effective medium of communication which explicitly represents the ideas and expressions of the human mind. It is the only channel by which human beings abstract reality. The importance of communication cannot be overemphasized and the need to express ideas, pass down knowledge and information among each other is a crucial part of living [2].

There are about 5000 languages spoken in the world today (a third of them in Africa). Despite decades of efforts on machine translations, fully-automatic general purpose high quality machine translation system (FGH-MT) is extremely difficult to build. In fact, there is no system in the world of any pair of languages which qualifies to be called a FGH-MT as affirmed in [4]. Machine translations of other countries such as European and Asian languages have been receiving considerable amount of research attention; but works on African Languages especially the Yorùbá language is rare [8].

NLP research is still in its infancy in Africa. In Nigeria alone there are about 729 languages, which have been categorized on the basis of the population of their speakers and relative importance into major, minor and minority languages. Hausa, Igbo and Yorùbá are the major languages, while languages such as Fula/Fulfude, Kanuri, Efik/Ibibio, Tiv and Izon are also categorized as constituting other class of major languages but smaller than those in the first category. The minor languages include Edo, Nupe, Urhobo, Igala, Idoma, Ebira, Itsekiri. While others not included in these minor groups are referred to as the minority languages. The languages spoken in Nigeria are not evenly distributed despite their large numbers. For instance in the South-West part of Nigeria, Yorùbá is largely spoken; Igbo is largely spoken in the South-East part of Nigeria; while in the North-West part of Nigeria, Hausa is largely spoken [2].
The dominance of the English language in Nigeria is quite enormous and this can be seen in virtually all sectors: government and administration, education, the media, the judiciary, science and technology to mention but a few. The major indigenous languages in the country are gradually slipping away because of the various trans-national structural revolutions in the name of globalization [4].

The need for translation is very glaring in the business community. It was observed that language barriers between organizations and their global customers are slowing down economic growth. According to a survey conducted in the United States, over $49 \%$ of company executives postulates that a language barrier has prevented a major international business deal and nearly 64\% of those same executives affirmed that language barriers are making it difficult to gain a foothold in International markets. In Nigeria, only few people speak more than one out of the three major indigenous languages and none of these languages is being elevated to the status of National language because of the general poor support for language research by the Nigerian Government.

Although, Yorùbá language is very rich in culture, it has been classified as a language in serious danger of extinction by the UNESCO Red Book on endangered languages as affirmed in [10]. The Yorùbá Language also known as (ede Yoruba) is spoken in three countries namely; Nigeria, Togo and Republic of Benin. The language is spoken in some communities in other parts of Africa, and it is even spoken outside the Africa continent as a great number of speakers of the language is found in Brazil, Cuba, Haiti, Caribbean Islands, Trinidad and Tobago, Europe and America. It is a tonal language with very little inflectional morphology and a strictly subject-verb-object (SVO) word order [2].

There is need to establish a basis for continuous machine translation research in the language in order to address some of its challenges and prevent the language from going into extinction. As a result of the syntax, structural and vocabulary differences in translations from English to Yorùbá and vice versa, translation works in the two languages requires a very good knowledge of the source language, the grammatical features of the target language, as well as familiarity with the subject matter of the text being translated [4].

In this research work, a user-friendly platform for easy text translation guide for Yorùbá terminologies used in building technology and their equivalents in English language has been developed. It is a unidirectional bilingual-lexicon of building technicians terminologies in which translation is from the Yorùbá language to English language. This system will contribute immensely to the reduction of language barrier and help in business transactions. This system aims to help improve and enhance communication skills of building technicians and to bridge communication gaps between individuals in this occupation field. This domain has been chosen because it is an area where language development is 
naturally taking place as new building materials with new names continue to evolve. This electronic lexicon provides translations to major terminologies in the field to enhance learning and communication.

\section{LITERATURE REVIEW}

In Nigeria, a lot of machine translation approaches has been employed by researchers in the automatic translations of English to Yorùbá language and vice-versa.

In [4], Web-Based English to Yorùbá Noun-Phrases Machine Translation System was developed. The research was carried out because of the need to address some of the challenges of English to Yorùbá language. Rule-based approach was used and the formulated rules were specified using context-free grammar. Finite state automata was used to formulate computational model and also for recognizing the grammar of the language.

A Yorùbá -English language translator for doctor-patient mobile chat was presented in [17]. They were motivated by the need to improve rural-urban health care by reducing communication barrier between semi illiterate patients and highly educated medical personnel who are of different ethnic background. Results show that the application has a high degree of novelty and relevance with about $60 \%$ and $80 \%$ scores respectively.

English to Yorùbá Bilingual Lexicon for Health sectors using direct approach to machine translation was developed in [3]. They were motivated by the need to address the issue of dissemination of information among people in health sectors and dwellers of some rural areas in Yorùbá land through the use of computers. It is a platform designed using direct approach to MT to provide easy to read guide for all diseases names and their various meanings in Yorùbá language. The system aimed at enhancing effective communication between medical practitioners and patients especially those in the southern rural environments.

[8] Presented English to Yorùbá Machine Translation System using rule-based approach. The motivation for the work was based on the need to contribute to knowledge in machine translations by experimenting with an African language. Text corpora were collected from home domain, context-free grammars were used to model the two languages, re-write rules and parse trees were also used. Automata theory was used to recognize the computational problem underlining the translation process.

A Statistical Machine Translation System for English and Nigeria Languages was developed in [6]. The motivation for the work was based on the need and urgency to increase the scale of research for the development of translation tools and devices for some languages due to the pressure of globalization. In the thesis, statistical approach to machine translations was used to set-up experiments on translation systems between English and two Nigerian languages: Igbo and Yorùbá. The study is setup to build parallel corpora, train and experiment English-to-Igbo, English-to-Yorùbá, and Igbo-to-Yorùbá, phrase-based statistical machine translation systems. The systems were trained on parallel corpora that were created for each language pair using text from the religious domain in the course of the research.

English to Yorùbá Statistical Machine Translation System was developed by [9]. The motivation for the work was due to the observation of Nigeria languages going towards total extinction. Phrase-based machine translation system was formulated to translate English to Yorùbá sentences and vice-versa. The system was implemented with Moses toolkits and evaluated with BLEU score.

All these researchers emphasized and encouraged the need for further research in Yorùbá language because of the need and urgency to increase the scale of research for the development of translation tools and devices for some languages especially the Yorùbá language due to the pressure of globalization.

\section{MATERIALS AND METHODS}

The system is a web-based application for Yorùbá to English translations for building technicians. The goal of the system is to reduce language barrier and promote the Yorùbá language by formulating another language resource for it through the development of a bilingual lexicon for building technicians. It is a unidirectional translation mode in which the direct-based approach to machine translations was adopted for the translations of Yorùbá to English terminologies. The system also includes an audio translation which allows users to listen to the pronunciation of any translated word.

The methods of study that were adopted in this research include: due consultations with language experts as well as the review of related literatures in both Yorùbá and English language. Data were collected from building technicians in Yorùbá language for a period of three months. Over 500 building terminologies: words and multi-words were gathered in Yorùbá language and translated to their equivalents in English language with the help language experts and linguistics for a period of four months. The programming language tools used were ASP. NET C\#, HTML, CSS, JavaScript and BOOTSTRAP as front end to provide a user friendly interface and Microsoft SQL (Structure Query Language) Server as backend to create the database for the data gathered.

\section{The Direct-Based Machine Translation Approach}

The Direct-based approach to machine translations in this work involves a unidirectional word for word translation of building technicians' terminologies from Yorùbá Language to English Language. In this system, a user types in a word in Yorùbá language and generates its equivalent meaning in 
English language. A user can also listen to an audio pronunciation of such word in English language to enhance learning. The architecture of the system is illustrated in Figure 1. The modules of the architecture are: source text, preprocessing, lexical transfer, target language syntactic generator and target output. Each module in the architecture is further explained:

\section{a. Source Text}

This is the first module in the architecture, when a user inputs a source text in Yorùbá Language into the system; the source text is accepted by the system and then enters the preprocessing stage.

\section{b. Preprocessing Stage}

At the preprocessing stage, the source text is first stored, after which it goes through preprocessing stage to determine the number of words in it. The system stops whenever a space is encountered, which signifies the end of a word and it eliminates the space automatically and moves to the lexical transfer stage.

\section{c. Lexical Transfer}

The outputs from the preprocessing stage enter lexical stage. At this stage, the corresponding target text equivalent is assigned to each word of the input text. This is achieved with the use of the bilingual lexicon made up of terminologies for building technicians in Yorùbá and English Language.

\section{d. Target Language Syntactic Generator}

The target text equivalent obtained for the source text in the lexical transfer phase enters this phase to produce the target text output.

\section{e. Target Text}

This module displays the output of the source text in English language.

f. Bilingual Lexicon: The bilingual-lexicon is the repository for Yorùbá and English Language building terminologies respectively.

The architecture is clearly illustrated in Figure 1.

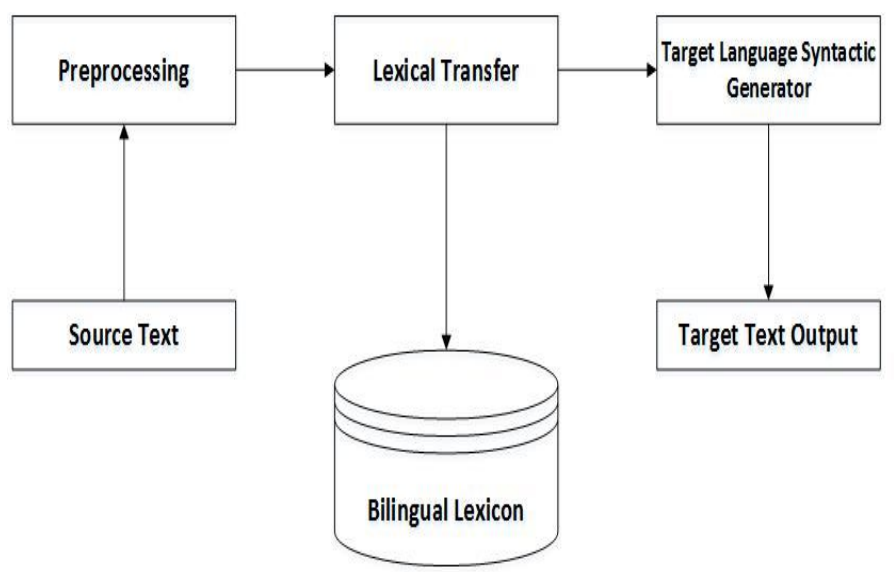

Figure 1: Architecture of the System

The operation of the system is activated when a user enters a Yorùbá language term into the system. The system goes through preprocessing to obtain the property of such word from the bilingual lexicon. If it is found, the English equivalent is obtained and displayed. The user can also listen to an audio pronunciation of the word (optional). However, if the equivalent of the source text is not found, a pop up message is displayed by the system to know if the user is willing to input another term. If yes, it returns to the start field, otherwise halts the process meaning that such input text is not in the repository. Samples of terminologies for building technicians in both languages are also displayed in Table 1.

Table 1: Some Terminologies for Building Technicians
TermsENG

Abrasion

Abrasive

Abut

Access Road

Accessory

Acqusition

Adaptor

Adhesion

Adhesive

Contact adhesive Gap-filling adhes...

Agent

AirConditioner

AirConditioning

Airtight

Align

Alignment

Aluminion roofing

Amount

Ampere

Amp

Angle fillet

Angle iron

Anteroom

Anti-termite Trea...

Apartment
Angle
TermsYOR iha-nnkan aha-nnkan fegbe kan titi atide afikun isoditemi alarina ilepo oole oole aganlaf... oole abuledi asoju amuletutu imuletutu asedemafefe isedemafefe iwanilakannaa ibole alumino iye aampiya aampu igun abudi eso oni... irin gbooro o... yara akokan oogun ikan ibudagbe

\section{RESULTS AND DISCUSSION}

The web-based Yorùbá to English bilingual lexicon for building technicians was implemented and tested to ensure accuracy and integrity in translations. The system consists of 
two major interfaces: the administrator view and the user's view. The user's view which is also the homepage can be accessed without any authentication. This view consists of the translation field where the technicians' terminologies in Yorùbá language are translated and their equivalents in English language are displayed. The system can also translate multi-word texts. The administrator view requires the admin to provide authentication to $\log$ in to the system. The Administrator view displays the lexicon used in the system and allows an admin to perform some action such as: adding new words, updating already existing words and deleting words from the lexicon as the case may be.

\section{Use Case Diagram}

The use case diagram is presented in Figure 2. The use case gives a simple diagrammatic representation of the user and administrator's interactions with the system. The administrator has access to the lexicon and can perform various operations on the lexicon such as; adding, updating and deleting. The user has access to the translation system and can perform translation operations such as translating building technicians' terminologies in Yorùbá language to English language and listening to the audio translation of translated terms.

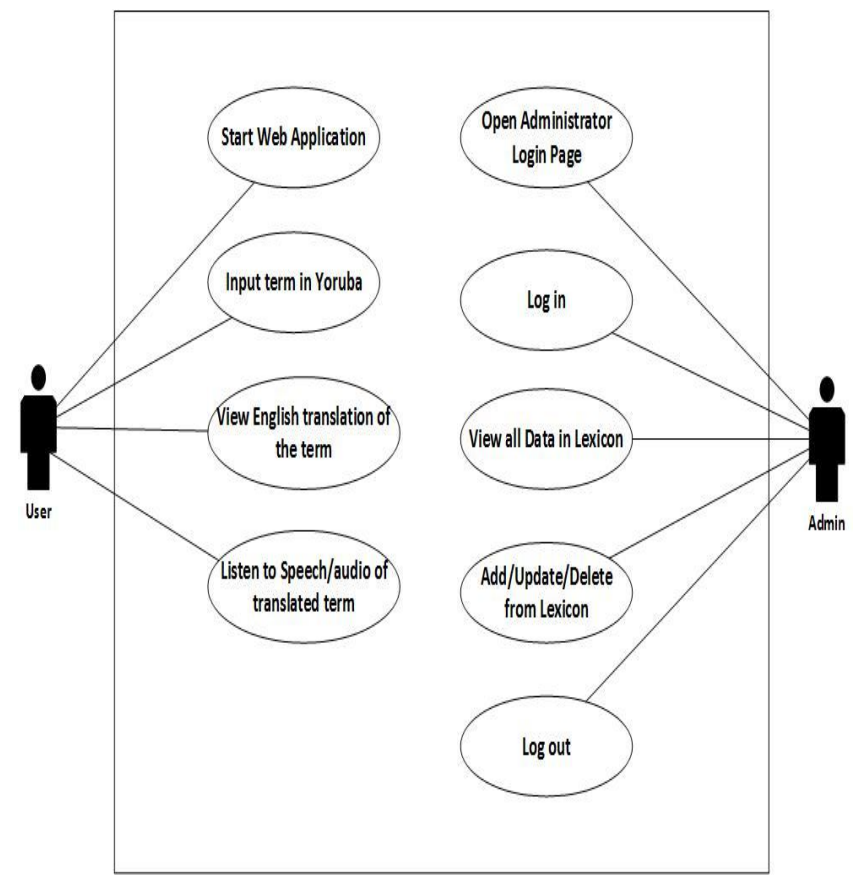

Figure 2: The Use Case Diagram

\section{The Home Page}

The homepage of the system is presented in Figure 3. It is the first interface that comes up when the system is launched. At the top right corner of the navigation bar, the Admin button is seen, this button when clicked shows the Administrator' Login Form.

\section{The Translation System Interface}

Figure 4 depicts the translation interface of the system. It consists of two text areas, Yorùbá language and English language. The field where texts are typed is the one named Yorùbá language. When a text is typed, the user proceeds to click the translate button after which the equivalent meaning in English language is displayed in the field with the caption English language.

A user can input a word and also input more than one word at a time as presented in Figure 4, where multiple words are entered into the system and their English equivalents are displayed. In Figure 4, we have "air conditioner, air-conditioning, lighting, power, sieve, soil" displayed for six different Yorùbá words that were entered into the Yorùbá language field for translations. Also, at the top right corner of the English language field, there is a button for the audio interpretation of an input text. A user can click on it for any English word to learn its pronunciation so as to enhance learning.

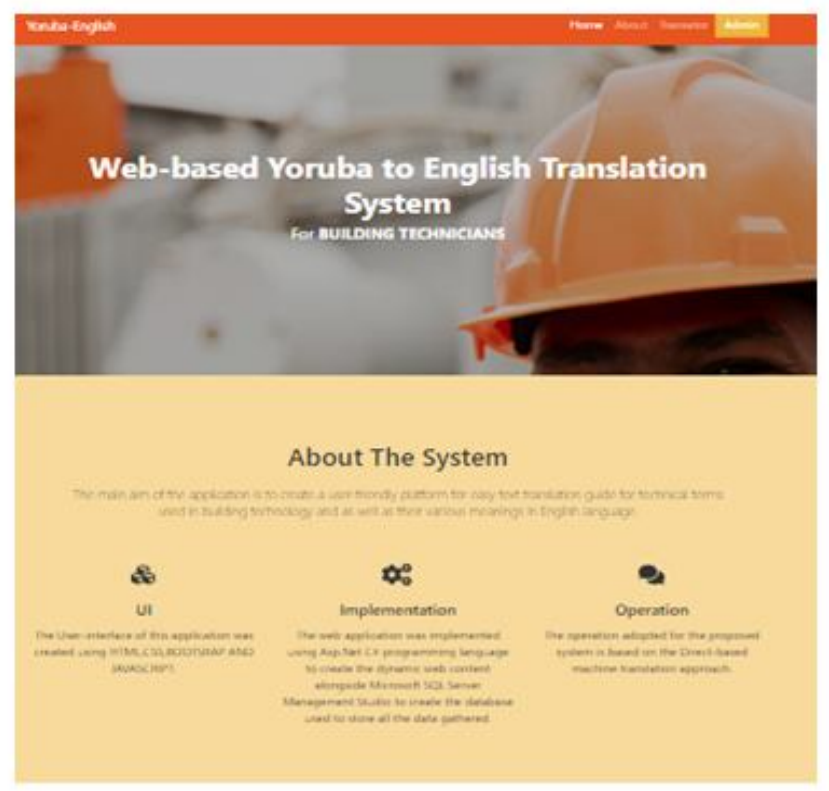

Figure 3: The Home Page

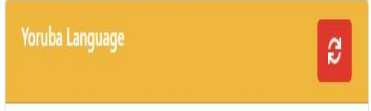

amuletutu, imuletutu, itanna, agbara, ajo,iyepe (n)

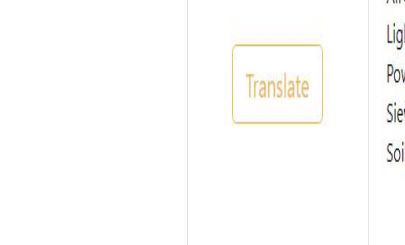

Figure 4: The Translation Interface 


\section{Administrator Login Interface}

The Administrator Login Form is presented in Figure 5 and it can be accessed by clicking the Admin button on the homepage. The Admin is required to login with username and password as shown in Figure 5. Here the admin can view all the data in the lexicon and can search through all the records. The Admin can also delete, update and add new word to the repository records.

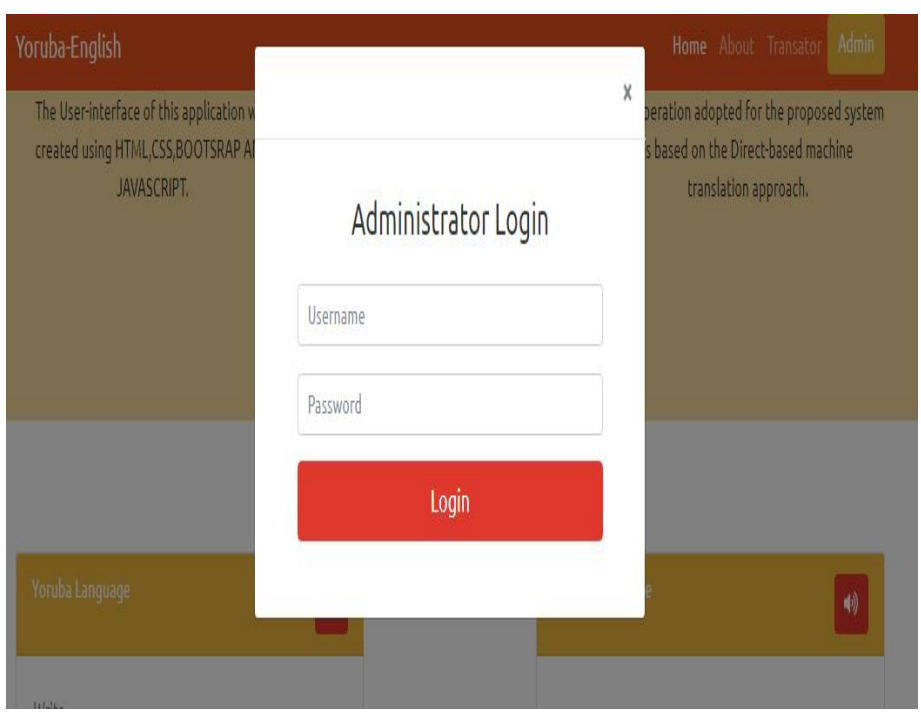

Figure 5: The Administrator Login Interface

\section{CONCLUSION AND RECOMMENDATION}

This research work has demonstrated the possibility of establishing a basis for continuous machine translation research in the Yorùbá language to address some of its challenges and prevent the language from going into extinction. This system will contribute immensely to the reduction of language barrier and help in business transactions for building technicians. The system will also help to improve and enhance communication skills of building technicians, bridge communication gaps between individuals in this occupation field. However, the research can be extended to accommodate more indigenous languages such as Igbo, Hausa and other minor languages in Nigeria, also translations can be provided for other artisans such as plumbers, fashion designers, mechanics and so on to enhance business transactions.

\section{REFERENCES}

[1] Ab́iọ́lá, O.B., Adétúńmbí A.O., \& Òguńtìmílệhìn, A. (2013).Computational Model of English To YorùbáNoun-Phrases Translation System. FUTA Journal of Research in Sciences, 9 (1): 34-43.

[2] Abiọ́lá, O.B., Adétúńmbí, A.O., \&Òguńtìmílệhìn, A. (2015).Using Hybrid Approach For English-to-Yorùbá Text to Text Machine Translation System (Proposed).
International Journal of Computer Science and Mobile Computing, 4 (8): 308-313.

[3] Abiola, O.B., Babalola, G.O. \& Adaramola O.O. (2015) An English to Yoruba Bilingual Lexicon for Health Sectors Using Direct Approach to Machine Translation. International Journal of Computer Science and Mobile Computing. 4(9): 29-57.

[4] Adéoyè, O.B. (2012). Web-Based English to Yorùbá Noun-Phrases Machine Translation System.M. Tech. Thesis, Federal University of Technology Akure.

[5] Amarpreet, K.,\& Er.Jyoti, R. (2014).A Review on a Web-Based Punjabi to Hindi Statistical Machine Translation System. International Journal of Advanced Research in Computer Science and Software Engineering (IJARCSSE), 4 (8): 383-386.

[6] Ayogu, I.I. (2018). Development of a Machine Translation System for English, Igbo andYorùbá Languages. Ph.D. Thesis, Federal University of Technology Akure

[7] Costa-Jussa, M.R., Farrus, M., Marino, J.B \& Fonollosa, J.A.R. (2012).Study and Comparison of Rule-Based and Statistical Catalan-Spanish Machine Translation Systems. International Journal of Computing and Informatics 31(2): 245-270.

[8] Elúdiórà, S.I. (2014). Development of anEnglish to YorùbáMachine Translation System. PhD Thesis, Obafemi Awolowo University, Ile-Ife.

[9] Fásakin, T.G. (2017). An English to Yorùbá Statistical Machine Translation System. M.Tech Thesis, Federal University of Technology, Akure.

[10] Francis, M.T. (2010). Rule-Based Breton to French Machine Translation. In European Association for Machine Translation.www.ofis-bzh/resources linguistigues/index-troerofis.php.Retrieved in 2015.

[11] Koehn, P., Och, F. J., \& Marcu, D. (2003).Statistical Phrase-Based Translation. In Proceedings of the 2003 Conference of the North American Chapter of the Association for Computational Linguistics on Human Language Technology, Volume1 (pp. 48-54).

https://doi.org/10.3115/1073445.1073462

[12] Mariana, N. (2016). Introduction to Natural Language Processing.IT system Engineering, Hasso Plattner Institute (HPI).

[13] Michel, S., Nicola, U., Pierre I., \& Roland K. (2007).Rule-Based Translation with Statistical Phrase-Based Post-Editing.In Proceedings of the Second Workshop on Statistical Machine Translation, (pp 203-206).

[14] Mohamed, A. E. (2000). Machine Translation of Noun Phrases from English to Arabic M.Sc. Thesis, Cairo University, Giza Egypt.

[15] Nakul, S. (2011).English to Hindi Statistical Machine Translation System, M.Sc. Thesis, Tharpa University Indian.Retrieved in 2015.

[16] Okpor, M.D. (2014). Machine Translation Approaches: Issues and Challenges. International Journal of Computer Science IJCSI, 11 (5): 159-165. 
[17] Oladosu, J.B., \& Olamoyegun. (2012). A multi-lingua expert system for rural $\mathrm{m}$-healthcare. International Journal of Electronic Healthcare, 7(2), pp 141-156.

https://doi.org/10.1504/IJEH.2012.049875

[18] Osborne, M. (2012).Machine Translation History and Rule-Based Systems. School of Informatics, University of Edinburgh.

[19] Sangeetha, J., Jothilakshmi, R.N., \& Devendra K. (2014).An Efficient Machine Translation System for English to Indian Languages Using Hybrid Mechanism. International Journal of Engineering and Technology (IJET), 6 (4): 1909-1919.

[20] Shaibu, S.D. (2013). Language Policy: Nigeria and the Role of English Language in the 21st Century. In European Scientific Journal, 9 (17): 1-21.

[21] Abdul, M.S., ByeongMan, K., \& HyunAh, L. (2019). English to Luganda SMT: Ganda Noun Class Prefix Segmentation for Enriched Machine Translation. International Journal of Advanced Trends in Computer Science and Engineering (IJATCSE), 8 (5): 1861-1868.

https://doi.org/10.30534/ijatcse/2019/08852019

[22] Nishad, N. (2019). Artificial Intelligence Interchange Human Intervention in the Recruitment Process in Indian Software Industry. International Journal of Advanced Trends in Computer Science and Engineering (IJATCSE), 8 (4): 1433-1442.

https://doi.org/10.30534/ijatcse/2019/62842019 